\title{
A Highly Accurate Model-Free Motion Control System with A Mamdani Fuzzy Feedback Controller combined with A TSK Fuzzy Feed-forward Controller
}

\author{
Qun Ren and Pascal Bigras \\ Department of automatic manufacturing Engineering \\ École de technologie supérieure, University of Quebec \\ 1100 Notre-Dame St W, Montreal, QC, Canada H3C 1K3 \\ qun.ren@polymtl.ca,pascal.bigras@etsmtl.ca
}

\begin{abstract}
In this paper, a new intelligent robot motion control architecture - a highly accurate model-free fuzzy motion control- is proposed in order to achieve improved robot motion accuracy and dynamic performance. Its architecture combines a Mamdani fuzzy proportional (P) and a conventional integral (I) plus derivative (D) controller for the feedback part of the system, and a TakagiSugeno-Kang fuzzy controller for the feed-forward, nonlinear part. The fuzzy P+ID controller improves the performance of the nonlinear system, and the TSK fuzzy controller uses a TSK fuzzy inference system based on extended subtractive- clustering method which integrates information on joint angular displacement, velocity and acceleration for torque identification. The advantage of this kind of model-free control is that it uses the information directly from the input/output of the nonlinear system, without any complex robot model computation, in order to decrease the control system's sensitivity to any dynamical uncertainty. Furthermore, parametric search for clustering parameters in extended subtractive clustering secures the high accuracy of the system identification. Consequently, this proposed model-free fuzzy motion control benefits from the advantages of two kinds of fuzzy system. It not only incorporates flexible design, good performance and simple conception but also ensures precise motion control and great robustness. Comparisons with other intelligent models and results from numerical studies on a 4-bar planar parallel mechanism show the effectiveness and competitiveness of the proposed control.
\end{abstract}

Key words - fuzzy systems, nonlinear dynamics, motion control, fuzzy logic control, model-free control, intelligent control

\section{Nomenclature:}

AI: $\quad$ artificial intelligence

AMFFMC accurate model-free fuzzy motion control

ANFIS adaptive network-based fuzzy inference system

FIS fuzzy inference system

FL: $\quad$ fuzzy logic

FLC: $\quad$ fuzzy logic control

FLS: $\quad$ fuzzy logic system

fuzzy P+ID fuzzy logic proportional plus conventional integral and derivative

MF: membership function

MF-PID-FLC model-free PID fuzzy feed forward control

MISO: multi-input-single-output

$\mathrm{NN}$ : neural network

PID: $\quad$ proportional, integral, derivative

RMSE: root-mean-square-error

TSK: $\quad$ Takagi-Sugeno-Kang 


\section{Introduction}

The development of new intelligent control techniques with nonlinear approximation capabilities, such as artificial neural networks (ANNs) [36], fuzzy logic systems (FLSs) [23, 37] called fuzzy logic controls (FLCs), and hybrid adaptive neurofuzzy inference systems (ANFISs) [1,2,13], has provided attractive alternatives in the identification of complex and essentially nonlinear systems. Intelligent controls are able to compensate for errors like dynamical uncertainty regardless of external disturbances caused by the outside environment. In the field of control, they are nonlinear mappings in the first place. Among them, FLCs $[24,39]$ have shown that such hybrid structures not only work well but also add more robustness to the control system's design. FLCs are able to deliver a satisfactory performance in the face of uncertainty and imprecision [9, 19]. Their suitability in nonlinear systems and in wide deviations from the set point has earned them their dominant position on the knowledge-rich spectrum of control algorithms. But most existing controls have to work with traditional conventional controls which are somehow dependent on human experience. The fuzzy rules in some of these are not directly generated from the robot's dynamics but depend solely on expert knowledge. Consequently, these kinds of control are sometimes unreliable [37, 38].

Intelligent PID controllers, found in the literature with different definitions [3], have been proposed for incorporation into model-free control of nonlinear systems that are difficult to identify with relative accuracy. According to Fliess and Join's paper [10], the key idea behind a model-free control is an ultra-local model instead of an unknown complex mathematical model. Existing model-free controls currently employ functional analysis, elementary differential algebra and online parameter identification approaches $[4,11,14,20,21,32,35]$. For example, iterative feedback tuning has been integrated into a PID controller to solve the controller tuning issues caused by model uncertainty of the nonlinear system [12]. Those existing fuzzy controls $[5,16,25,31]$ aim at tuning controller parameters for the nonlinear system whose model is difficult to identify with relative accuracy through different mathematical identification of the system approaches. But these existing controls have fuzzy rules in the intelligent PID controllers which depend solely on expert knowledge. Moreover, mathematical identification approaches are unsuited to the non-deterministic nature of several parts of robotic systems.

The objective of this paper is to achieve the high accuracy tracking control of an unknown nonlinear robot system, using a novel intelligent robot motion control - an accurate model-free fuzzy motion control (AMFFMC). The proposed AMFFMC is different from existing intelligent and model-free controls. This kind of control combines a hybrid Mamdani fuzzy proportional with a conventional integral plus derivative (fuzzy P+ID) controller [15] for the feedback part of the system, and a TakagiSugeno-Kang (TSK) fuzzy controller [28] based on extended subtractive clustering [29] for the feed-forward nonlinear part. This novel control not only has flexible design, good performance and simple conception but also ensures stable control quality and great robustness against disturbance effects in real world applications. The fuzzy P+ID control improves the dynamic response of the system, without using the information of the nonlinear system, which is necessary for accuracy. The highly accurate system identification model for the TSK FLC is constructed through training a FLS by integrating the robot's actual joint angular displacement $(p)$, angular velocity $(v)$, angular acceleration, $(a)$ and joint torque $(\tau)$ data set, using an extended subtractive clustering technique. This kind of control acknowledges information directly from the nonlinear dynamics of the physical robot, without any complex robot model computation. The results of a numerical simulation and comparisons made on a planar 4-bar parallel mechanism show the effectiveness and satisfactory performance of the AMFFMC.

In the remainder of this paper, a brief description of the fundamentals of FLCs and extended subtractive- clustering are given in Section 2. The proposed AMFFMC is presented in Section 3. Details of the AMFFMC design schemes are in Section 4. The numerical comparison of the proposed control and its sub-controls are in Section 5 to show its advantages and efficiency. Finally, the concluding remarks and future research recommendations are in Section 6. 


\section{Theoretical Foundation}

\subsection{Fuzzy Logic Control}

The two most important classes of fuzzy logic system (FLS) used by today's engineers are the Mamdani FLS and the TSK FLS. Moreover, fuzzy logic control (FLC) and its well- accepted methodology for designing controllers is the most widely used application of fuzzy logic (FL)

Mamdani was the first to apply fuzzy logic to control [18]. This topic has come to be known as fuzzy algorithmic control or linguistic control. The main problem, however, with fuzzy control is the designing of a fuzzy controller, where we usually take an expert-system-like approach. That is, we derive the fuzzy control rules from the human operator's experience and/or the engineer's knowledge, which are mostly based on their qualitative knowledge of an objective system.

The rules in a Mamdani fuzzy expert system are usually of a form similar to the following:

$$
\begin{aligned}
& \text { IF } x_{1} \text { is } Q_{1} \text { and } x_{2} \text { is } Q_{2}, \\
& \text { THEN } Z \text { is } w
\end{aligned}
$$

where $x_{1}$ and $x_{2}$ are linguistic input variables, $Z$ is a linguistic output variable. $Q_{1}$ and $Q_{2}$ are linguistic values defined by fuzzy sets on the universes of discourse $X_{1}$ and $X_{2}$.

Subsequently, the TSK FLS [22, 30, 33, 34] was proposed as a further qualitative modeling in an effort to develop a systematic approach to generating fuzzy rules from a given input-output data set. This model consists of rules with fuzzy antecedents and a mathematical function in the consequent part. Usually the conclusion function is in the form of dynamic linear equation. The antecedents divide the input space into a set of fuzzy regions, while the consequents describe the behaviour of the system in those regions. But there is a need to develop a semi-automatic method to obtain these models, based on sets of inputoutput data.

For a multi-input single-output (MISO) first-order generalized TSK FLS, its $k^{\text {th }}$ rule can be expressed as:

$$
\begin{aligned}
& \text { IF } x_{1} \text { is } Q_{1 k} \text { and } x_{2} \text { is } Q_{2 k} \text { and } \ldots \text { and } x_{n} \text { is } Q_{n k} \text {, } \\
& \text { THEN Z is } w^{k}=p_{0}^{k}+p_{1}^{k} x_{1}+p_{2}^{k} x_{2}+\ldots+p_{n}^{k} x_{n}
\end{aligned}
$$

where $x_{1}, x_{2} \ldots, x_{n}$ and $\mathrm{Z}$ are linguistic variables; $Q_{1 k}, Q_{2 k}, \ldots$, and $Q_{n k}$ are the fuzzy sets on universe of discourses $\mathrm{U}, \mathrm{V}, \ldots$, and $\mathrm{W}$, and $p_{0}^{k}, p_{1}^{k}, p_{2}^{k}, \ldots, p_{n}^{k}$ are regression parameters.

Both Mamdani and TSK FLC systems are characterized by IF-THEN rules and have the same antecedent structures. However, there are some differences between them. First, the structure of the consequents for a TSK rule is a function instead of a fuzzy set as in a Mamdani rule. Second, the output of a TSK FLS is a crisp value, whereas the output of a Mamdani FLS is a fuzzy set; defuzzication is needed to obtain the crisp output by using the composition operator. Third, uncertainty can be accounted for in both the antecedent and consequent MFs in a Mamdani FLS, but in a TSK FLS, only in the antecedent MFs. To reiterate, Mamdani FLSs are intuitive and well- suited to human input whereasTSK FLSs are computationally effective but lose linguistic interpretability. They work well with optimisation and adaptive techniques, which makes them very attractive in solving control problems, particularly in dynamic nonlinear systems.

Fuzzy Logic Control (FLC) has proven to be a successful control approach to many complex nonlinear systems or even nonanalytic systems $[8,17]$. The basic structure of a fuzzy control system consists of four conceptual components: the knowledge base (fuzzy rule), the fuzzification interface, the inference system, and the defuzzification interface. Fig. 1 shows a typical FLC block diagram. The physical robot's input and output variables are a crisp number. However, inside the fuzzy control, the input and output variables are fuzzy numbers. 


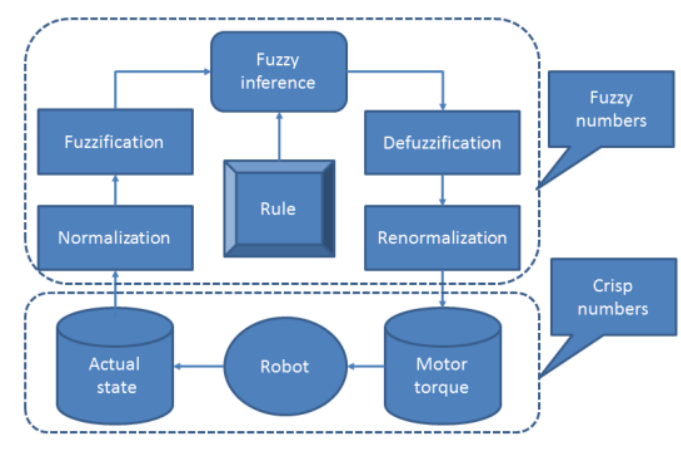

Fig. 1 Fuzzy logic control block diagram

\subsection{Extended Subtractive Clustering}

The generality of the TSK FLSs makes data driven identification very complex. In this paper, extended subtractive -clustering [26] is used for fuzzy system structure identification and least square estimation is used for parameter identification. The former is the determination of the number of rules and variables involved in the rule premises, while the latter is the estimation of the membership function parameters and the estimation of the consequent regression coefficients.

Chui's subtractive-clustering method [7] assumes that each data point is a potential cluster center and calculates a measure of the likelihood that each data point would therefore define the cluster center, based on the density of the surrounding data points. The four clustering parameters- hypersphere cluster radius, squash factor, accept ratio and reject ratio, have great inference on the number of clusters and the number of training iterations to be employed. They should be defined as parameters in the configuration of the fuzzy inference system (FIS). As a result of parametric search in Demirli's extended subtractive clustering [6], ranges of clustering parameters that provide the best models are also identified.

The recommended values from Chiu and Demerli of these four clustering parameters are listed in TABLE I. According to Demirli, parametric searches have to be done for all four clustering parameters, and the search range for cluster parameters is much larger than that of Chiu.

TABLE I

RECOMMENDED VALUES FOR PARAMETERS IN SUBTRACTIVE CLUSTERING

\begin{tabular}{|l|l|l|}
\hline Symbol & Chui [36] & Demirli [37] \\
\hline Cluster radius & {$[0.25,0.50]$} & {$[0.15,1]$} \\
\hline Squash factor & 1.25 & {$[0.05,2]$} \\
\hline Reject ratio & 0.15 & {$[0,0.9]$} \\
\hline Accept ratio & 0.5 & {$[0,1]$} \\
\hline
\end{tabular}

The extended subtractive-clustering algorithm is depicted in Fig. 2.A detailed description can be found in [27]. 


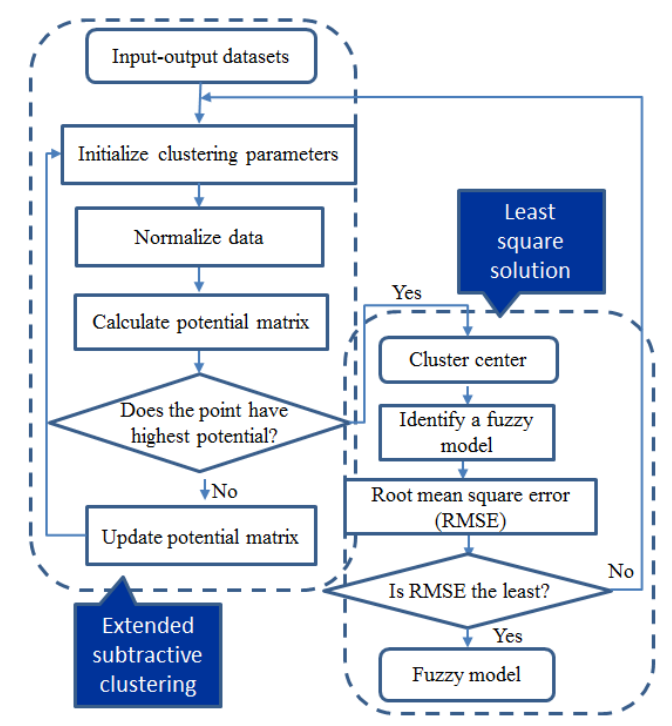

Fig. 2 Extended subtractive-clustering algorithm

\section{The Accurate Model-Free Fuzzy Motion Control}

\subsection{The Accurate Model-Free Fuzzy Motion Control}

The proposed control system, an AMFFMC is a combination of a Mamdani fuzzy P+ID feedback control [25] and a TSK fuzzy feed-forward control system. Its control law is written as

$$
\tau=K p u_{f u z z y}+K d\left(v^{d}-v\right)+K i \int\left(p^{d}-p\right) d t+\tau_{T S K}
$$

The three controller parameters $K p, K i$ and $K d$ are the proportional, integral, and derivative gains; $p^{d}$ and $v^{d}$ are the desired joint position and velocity; $t$ is the running time and $u_{f u z z y}$ is the Mamdani FLS output. $\tau_{T S K}$ is the TSK FLS output.

In comparison with existing intelligent controllers, the proposed AMFFMC combines the advantages of both the fuzzy P+ID control and the TSK fuzzy control. In the fuzzy P+ID control, the fuzzy P term improves the performances of nonlinear systems. The conventional integral I is responsible for the reduction of steady-state error, and the conventional derivative D predicts system behaviour and thus improves settling time and stability. In TSK fuzzy control, the precision of the system is greatly improved by using extended subtractive clustering for the TSK fuzzy system's pre-identification [27].

Furthermore, this controller has the following features:

1) Once $K p, K i$ and $K d$ are determined, only one additional parameter - the output of the fuzzy P system - has to be adjusted to achieve the fuzzy P+ID control. Moreover, the MF-PID-FLC is generated directly from the experimental data set ( $p, v, a$ and $\tau$ ). These FLCs are developed with no knowledge of the mathematical model of the robot being controlled. Thus, the AMFFMC is easy to design.

2) The AMFFMC has a simple structure. It is not necessary to modify any hardware parts of the original control system for its implementation.

3) This AMFFMC, with its simple conception - that of a fuzzy P+ID control for the feedback part of the system and a MFPID-FLC based on extended subtractive clustering for the accurate nonlinear part of the system guarantees accurate control and robustness against disturbance effects in real world applications. 


\subsection{The Mamdani Feedback Controller}

The Mamdani feedback controller is a fuzzy P+ID controller which combines fuzzy logic and conventional techniques. It generally provides better control performances than a PID controller alone. A hybrid fuzzy P+ID controller is designed by using a fuzzy logic controller in place of the proportional term, while the integral and derivative terms are kept unchanged.

From the expression (1), the fuzzy rules of this Mamdani FLC are described in terms of the input variables $e_{p}$ and $e_{v}$ and the output variable $u_{f u z z y} ; e_{p}$ and $e_{v}$ are the robot's position and velocity tracking error. The $k^{\text {th }}$ rule can be written as

$$
\begin{aligned}
& \text { IF } e_{p} \text { is } x_{k} \text { and } e_{v} \text { is } y_{k} \\
& \text { THEN } u_{f u z z y} \text { is } z_{k},
\end{aligned}
$$

where $x, y, z$ are the fuzzy subsets of inputs $e_{p}, e_{v}$ and output $u_{f u z z y}$. The MAX-MIN method with the MAX aggregation operator and the MIN AND operator are used as inference engines. The defuzzification method is the "center of mass".

The control law for the fuzzy P+ID feedback controller is

$$
\tau=K p u_{f u z z y}+K d\left(v^{d}-v\right)+K i \int\left(p^{d}-p\right) d t
$$

A detailed description of the fuzzy P+ID control can be found in [25].

\subsection{The Takagi-Sugeno-Kang- Fuzzy Feed- Forward Controller}

A fuzzy learning system based on the extended subtractive-clustering algorithm in Fig. 2 is used to integrate the information of $p, v, a$ and $\tau$ (the position, velocity, acceleration and torque of the active robot joints), and pre-identify a reliable FIS. From the expression (2), the expression of the $k^{\text {th }}$ fuzzy rules for this TSK fuzzy feed forward controller can be written as

$$
\begin{aligned}
& \text { IF } p \text { is } Q_{p k} \text { and } v \text { is } Q_{v k} \text { and } a \text { is } Q_{a k}, \\
& \text { THEN } \tau_{T S K} \text { is } \tau^{k}=f_{T S K}(p, v, a) \text { or } \\
& \tau^{k}=p_{0}^{k}+p_{1}^{k} p+p_{2}^{k} v+p_{3}^{k} a
\end{aligned}
$$

where $Q_{p k}, Q_{v k}$ and $Q_{a k}$ are the MFs in the $k^{\text {th }}$ rule associated with $p, v$ and $a$. The output $\tau$ is a polynomial linear function of $p$, $v$ and $a$.

Using the generated TSK FIS, a Model-free PID TSK fuzzy logic controller (MF-PID-FLC) is then conceived. Its control law can be written as

$$
\tau=K p\left(p^{d}-p\right)+K d\left(v^{d}-v\right)+K i \int\left(p^{d}-p\right) d t+\tau_{T S K}
$$

where $\tau_{T S K}$ is a polynomial function of $p^{d}, v^{d}$ and $a^{d}$, where $a^{d}$ is the desired joint acceleration :

$$
\tau_{T S K}=f_{T S K}\left(p^{d}, v^{d}, a^{d}\right) .
$$

A detailed description of the TSK fuzzy control system in the MF-PID-FLC can be found in [26].

\section{The Numerical Study}

4.1 The Simulink Model

Simulating robot motions allows the testing of control strategies and provides an insight into motion planning techniques without the need of a physically available system. The parallel robot torque control study was conducted on a planar quadrilateral 
linkage. The kinematics and dynamics of planar four-bar linkages are simpler but similar to other more-than-four-bar parallel robots. The parallelogram four-bar mechanism is a drag link with four rotating joints, as depicted in Fig 3 . The initial parameters are used only for establishing the Matlab Simulink mechanical system model. None of them will be used in the design of the proposed control.

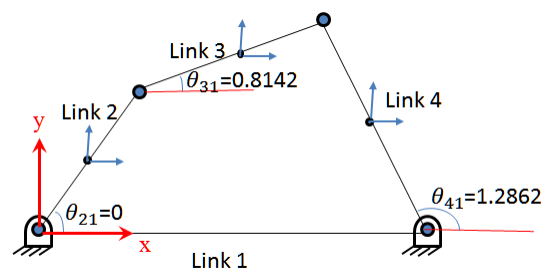

Fig. 3. Four-bar mechanism used for experimental study

\section{The Fuzzy P+ID Controller}

The fuzzy P system consists of a Mamdani type of inference system with two inputs $e_{p}$ and $e_{v}$ and one output $u_{f u z z y}$. The inputs $e_{p}$ and $e_{v}$ are the active joint position and velocity tracking errors. They are ranged by $e_{p} \in[-0.010 .01]$ and $e_{v} \in[-0.10 .1]$ based on the simulation result from the PID feedback control system, whose control law is written as

$$
\tau=K p\left(p^{d}-p\right)+K d\left(v^{d}-v\right)+K i \int\left(p^{d}-p\right) d t
$$

To be consistent with the input range, the $u_{f u z z y}\left(e_{p}, e_{v}\right)$ is ranged by $u_{f u z z y} \in[-0.010 .01]$.

There were a total of nine rules between inputs and output that are summarized in the following TABLE 2 .

TABLE 2

FUZZY RULES IN THE FUZZY P+ID CONTROLLER

\begin{tabular}{|c|c|c|c|}
\hline $\begin{array}{l}u_{f u z z y} \\
e_{v}\end{array}$ & negative & zero & positive \\
\hline negative & negative & negative & zero \\
\hline zero & negative & zero & positive \\
\hline positive & zero & positive & positive \\
\hline
\end{tabular}

The control diagram block is shown in Fig. 4.

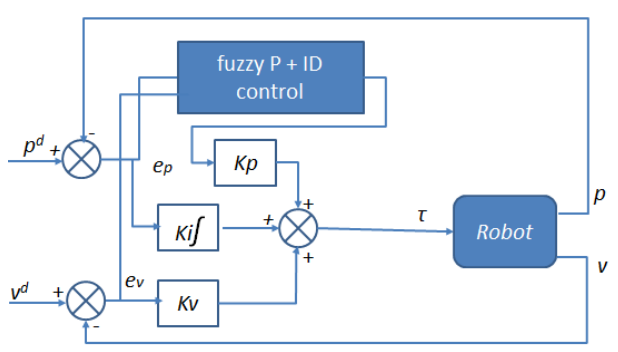

Fig. 4 Block diagram of the fuzzy P+ID controller 


\subsection{The Model-free PID Fuzzy Logic Controller}

Using a dynamic model to solve motion simulation requires the knowledge of the values of each dynamic parameter used in the model.

The PID feedback control law used in the training is written as

$$
\tau=K p\left(p^{d}-p\right)-K d(v)+K i \int\left(p^{d}-p\right) d t
$$

This control law was used to generate the experimental data set $(p, v, a$ and $\tau)$ for the analysis of the system, and to train the fuzzy rules for the fuzzy control. The desired position trajectory was a composite of multiple step signals, which were as rich as possible to allow the learning process of the TSK FIS to include all the characteristics of the nonlinear dynamics of the system. This step attempts to obtain motion data sets directly from the parallel mechanism that encounters manufacturing tolerances and assembly errors, without any calibration, in order to identify a precise intelligent control in the next step and decrease the sensitivity of the control system to any dynamical uncertainties whatsoever.

The data sets used for training were collected during 25 seconds, as depicted in the following Fig. 5. The experimental data set $(p, v, a$ and $\tau$ ) contained 10001 samples of data corresponding to a sampling time of $0.005 \mathrm{~s}$. The first three parameters were used as fuzzy identification input variables and the torque as the output variable. The system to be learned is thus the inverse of the robot model, which is an appropriate feed-forward controller that predicts the input of the system corresponding to the desired trajectory.

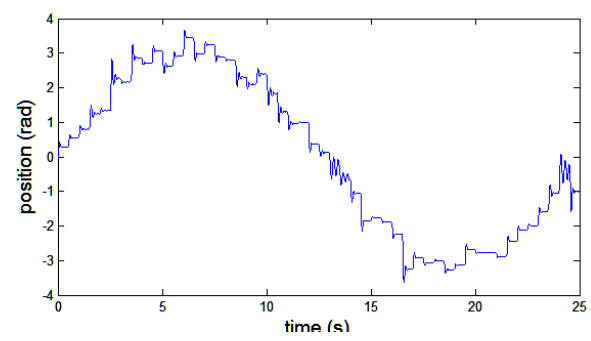

(a) Angular displacement (position $p$ )

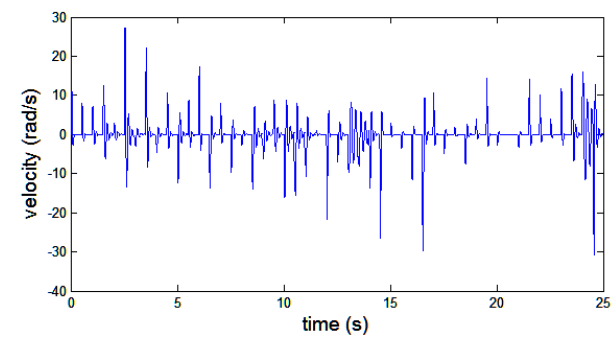

(b) Angular velocity (velocity $v$ )

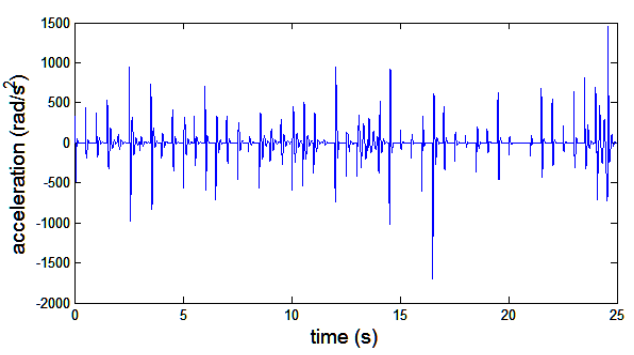

(c) Angular acceleration (acceleration $a$ ) 


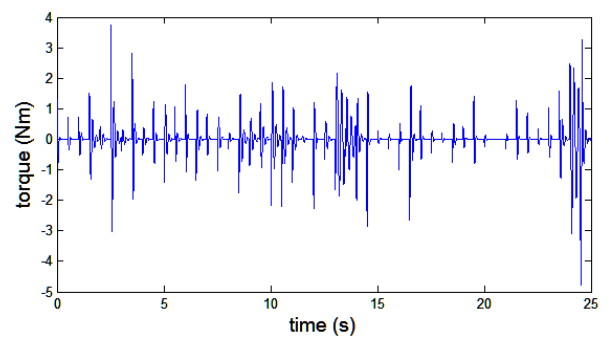

(d) Torque (torque $\tau$ )

Fig. 5. Fuzzy learning data

By the extended subtractive clustering based TSK fuzzy modelling algorithm, a fuzzy model with 96 fuzzy rules is generated. The parameters used in this FIS (hypershere cluster radius, squash factor, accept ratio and reject ratio) are 0.25, 0.6, 0.6 and 0.15. There are 96 MFs for each input variable. They are indicated in Fig 6. The identified fuzzy learning outputs are shown in Fig. 7. Fuzzy learning root-mean-square-error (RMSE) is 0.0025.
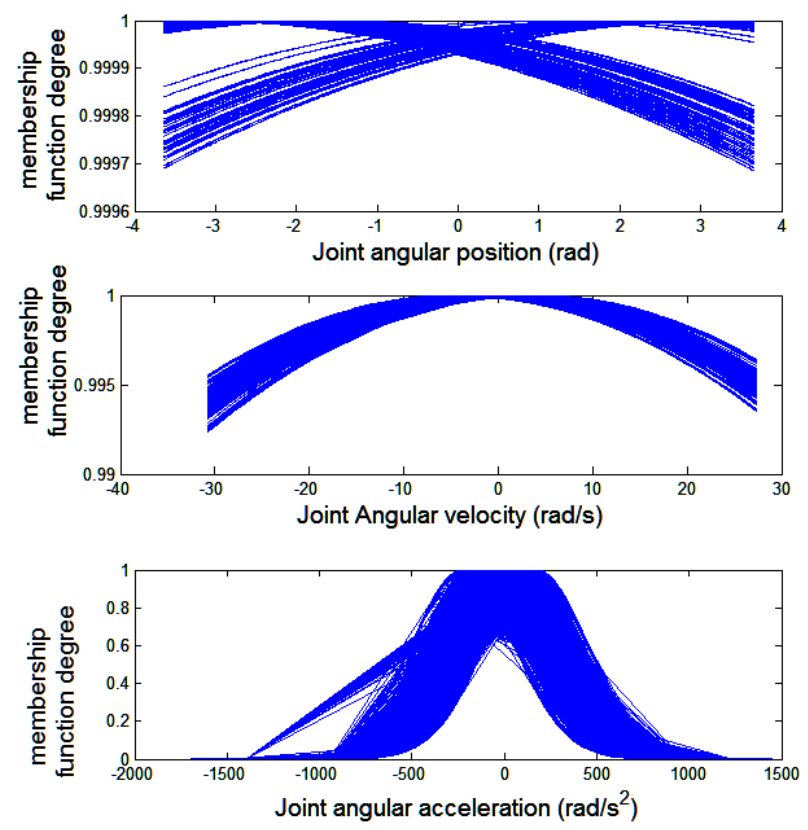

Fig. 6 Membership functions 


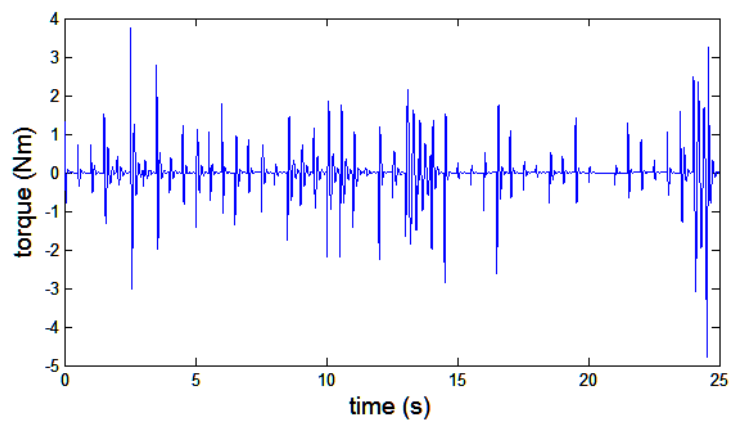

Fig.7 Fuzzy learning output

One example of the 96 fuzzy rules is given as follows:

$$
\begin{aligned}
& \text { IF } Q_{p}=\exp \left(-\frac{1}{2}\left(\frac{p+2.6809}{0.644}\right)\right) \\
& Q_{v}=\exp \left(-\frac{1}{2}\left(\frac{v+0.0243}{5.1245}\right)\right) \text { and } \\
& Q_{a}=\exp \left(-\frac{1}{2}\left(\frac{a+1.8487}{278.0148}\right)\right) \\
& \quad \text { THEN } \\
& \tau=6.7013 e-4 * p-9.04 e-6 * v+0.30 * a-0.037
\end{aligned}
$$

The 96 MFs for each input, the fuzzy learning outputs and the FIS output surfaces can be found in [24] The block diagram of the MF-PID-FLC used for simulation is shown in Fig. 6.

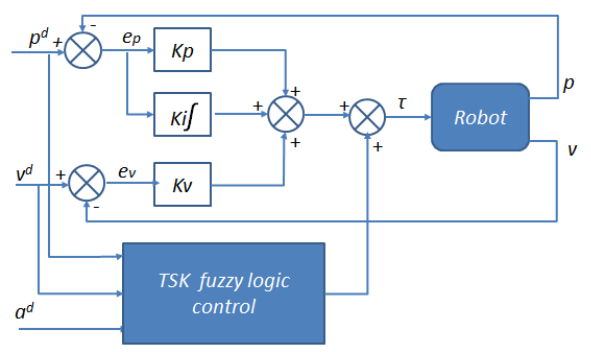

Fig. 8 Block diagram of the MF-PID-FLC

\subsection{An Accurate Model-Free Fuzzy Motion Control}

The block diagram of the proposed AMFFMC is shown in Fig. 9 below with the control law as the expression (2). Compared with Fig. 7, the position error $e_{p}$ is replaced by the output of the fuzzy P+ID control $u_{f u z z y}$. The inference engines of the fuzzy P+ID control and the MF-PID-FLC provide a set of control actions according to the fuzzified inputs. 


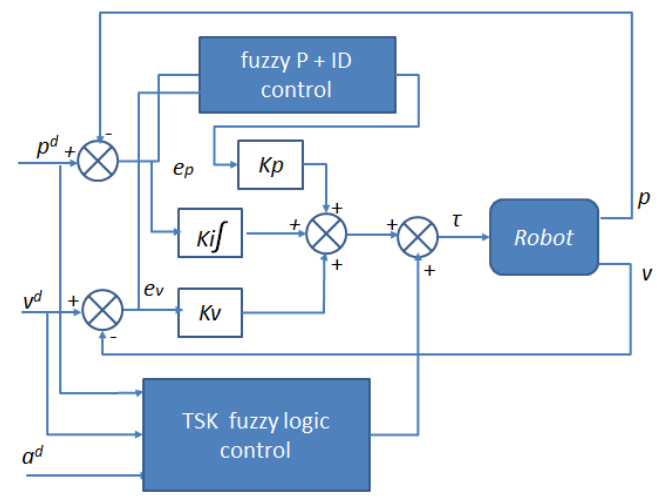

Fig. 9 Block diagram of the AMFFMC

To investigate the robustness, stability and tracking error convergence of the proposed AMFFMC, three desired motion trajectories, as listed in TABLE III, were used to test the novel trajectory control scheme.

The testing procedure used $p^{d}, v^{d}$ and $a^{d}$ as input data sets to compute the fuzzy output - torque values in the three different cases. Each experimental data set contained 2001 samples of data, corresponding to a sampling rate of $0.005 \mathrm{~s}$. TABLE III shows both the position and velocity tracking errors in the last five seconds using the three desired motion trajectories. From the last two lines, it is observed that, overall, the $e_{p}$ is smaller than 1.4e-3. and $e_{v}$ is smaller than 0.09 .

TABLE III

FuZZY MODEL TESTING DYNAMICS AND ERRORs

\begin{tabular}{|c|l|l|l|l|}
\hline \multicolumn{2}{|c|}{} & \multicolumn{3}{|c|}{ Testing } \\
\cline { 3 - 5 } & Test 1 & Test 2 & Test 3 \\
\hline \multirow{4}{*}{ Desired } & Position & $p^{d}=1-\cos (8 t)$ & $p^{d}=1-\cos (4 t)$ & $p^{d}=\sin (t)$ \\
\cline { 2 - 5 } & Velocity & $v^{d}=8 * \sin (8 t)$ & $v^{d}=4 * \sin (4 t)$ & $v^{d}=\cos (t)$ \\
\cline { 2 - 5 } & Accele & & & \\
& -ration & $a^{d}=64 * \cos (8 t)$ & $a^{d}=16^{*} \cos (4 t)$ & $a^{d}=-\sin (t)$ \\
\hline \multicolumn{2}{|c|}{ Position error } & {$[-6.41 \mathrm{e}-4$} & {$[-2.67 \mathrm{e}-3$} & {$[-6.41 \mathrm{e}-4$} \\
interval (rad) & $-1.17 \mathrm{e}-4]$ & $-7.47 \mathrm{e}-5]$ & $4.53 \mathrm{e}-4]$ \\
\hline \multicolumn{2}{|c|}{ Velocity error } & {$[-6.41 \mathrm{e}-4$} & {$[-1.17 \mathrm{e}-2$} & {$[-6.49 \mathrm{e}-3$} \\
interval (rad/s) & $7.42 \mathrm{e}-3]$ & $4.79 \mathrm{e}-3]$ & $3.07 \mathrm{e}-3]$ \\
\hline
\end{tabular}

5. Comparisons and Discussions

\subsection{Comparisons}

For an in-depth analysis of the proposed AMFFMC to show its advantages and capability, one desired joint trajectory $\left(p^{d}=1\right.$ $\cos (8 t), v^{d}=8 * \sin (8 t)$ and $\left.a^{d}=64 * \cos (8 t)\right)$ was used to compare the performance of the AMFFMC ( $e_{p}$ and $\left.e_{v}\right)$ with that of its subcontrols: the PID feedback control, the fuzzy P+ID control, and the MF-PID-FLC, with their controls listed as eq.(10), eq.(9) and eq.(5). The PID gains always always remained the same.

The position and velocity tracking errors $\left(e_{p}\right.$ and $\left.e_{v}\right)$ are compared in Fig. 8 and Fig. 9. The solid blue curves in Fig. 8, are the $e_{p}$ and $e_{v}$ of the system under AMFFMC. The dotted black curve, the dashed red curve and the dashed green curve are under fuzzy P+ID control, MF-PID-FLC and PID control. It is clear that the AMFFMC ensures the smallest error and results in the best performance. These curves also show that the fuzzy P+ID controller without fuzzy feed-forward is better than the traditional PID controller without fuzzy feed-forward. 


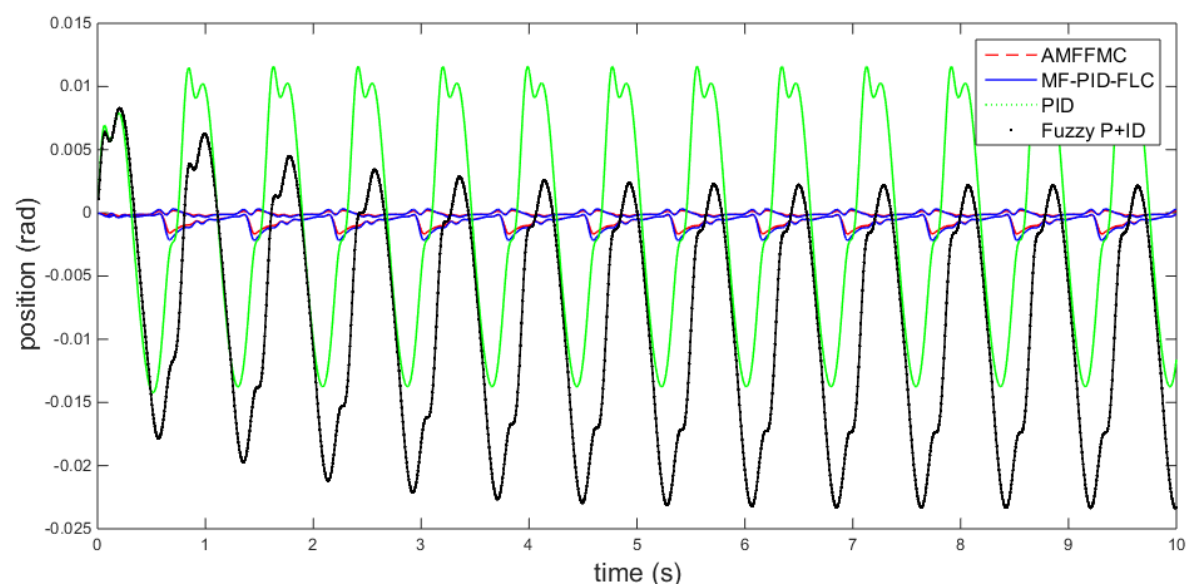

(a) Position tracking errors

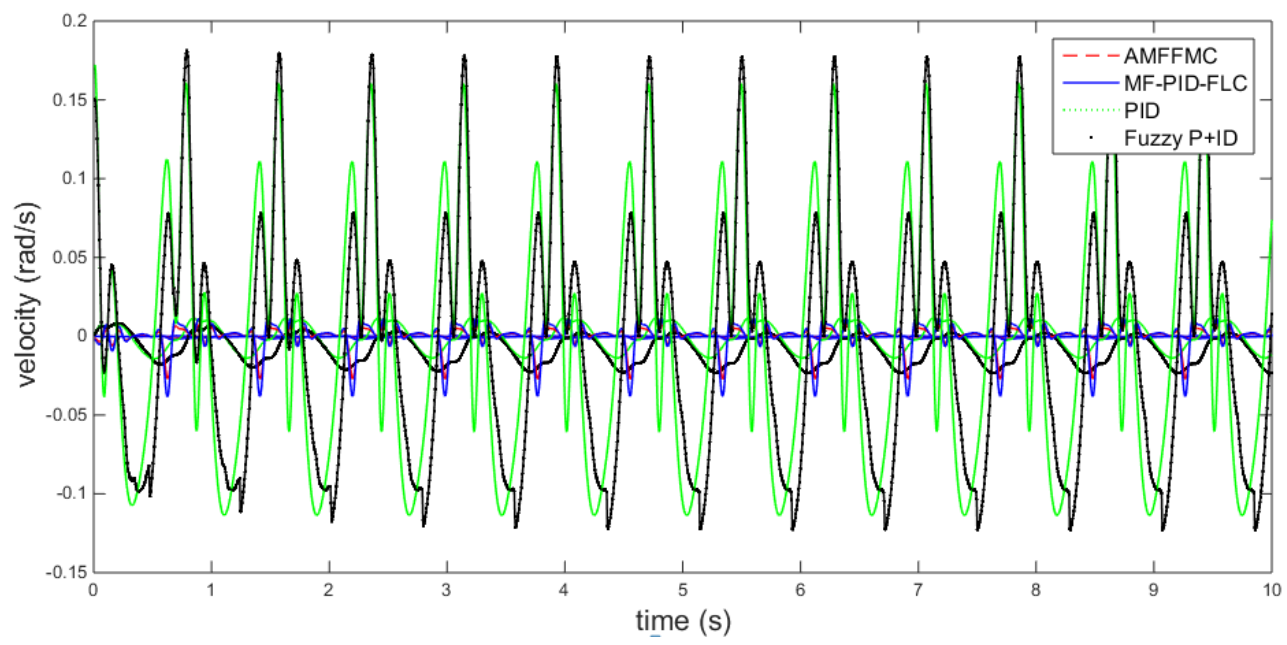

(b) Velocity tracking errors

Fig. 10 Position and velocity errors $p^{d}=1-\cos (8 t)$

In order to analyse the results accurately, the worst positive and negative errors during the last 5 seconds are compared in Fig. 9. It clearly shows the differences between the AMFFMC and its sub-controls.

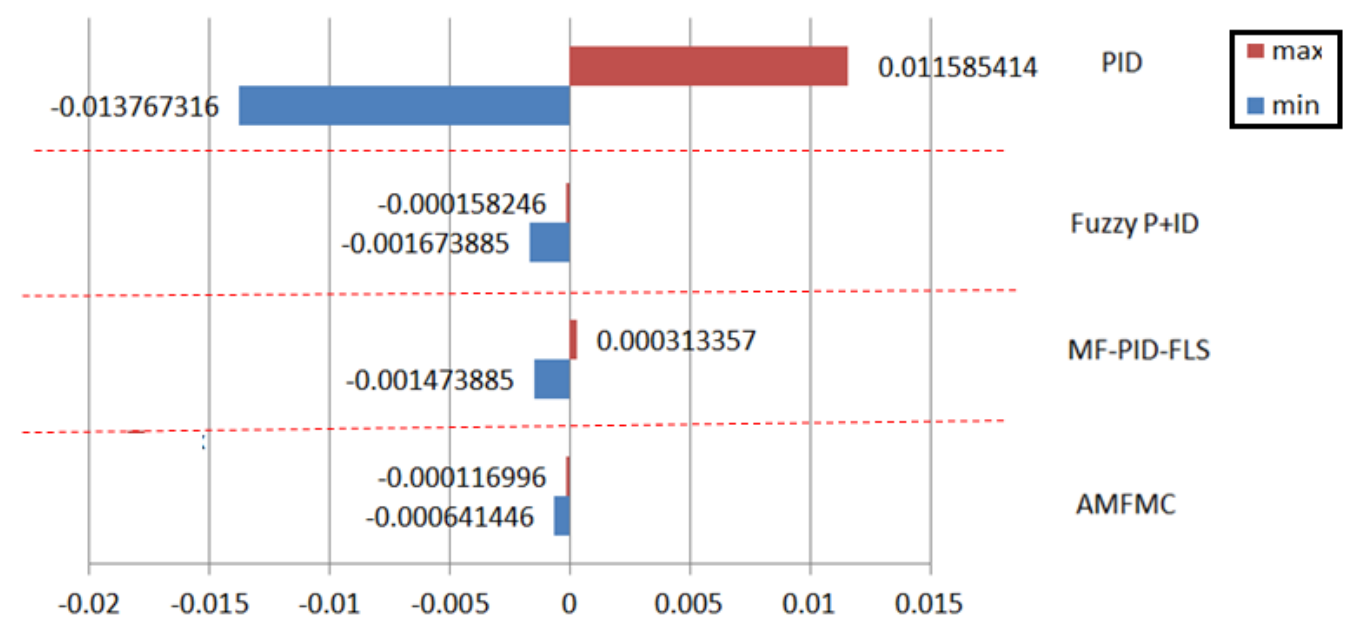

(a) Position tracking error interval values when $p^{d}=1-\cos (8 t)$ 


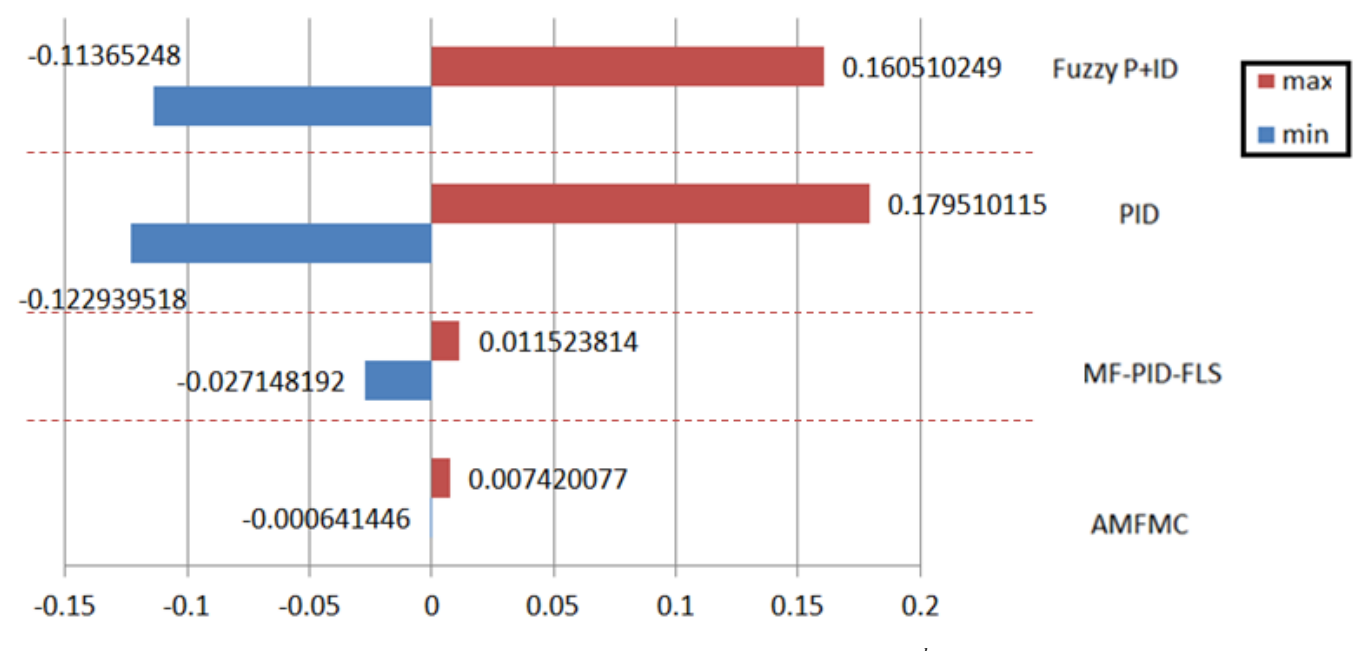

(b) Velocity tracking error interval values when $p^{d}=1-\cos (8 t)$

Fig. 11 Position tracking error interval values

In position tracking, the AMFFMC performs more than 2.5 times better than the MF-PID-FLC, 26 times better than the PID control and 25 times better than the Fuzzy P+ID control. In velocity tracking, the AMFFMC performs more than 1.7 times better than the MF-PID-FLC, 20 times better than the PID and 18 times better than the Fuzzy P+ID. It has been shown, therefore, that the AMFFMC has the capability of reducing the tracking errors, and providing higher accuracy and greater reliability in controlling a nonlinear parallel mechanism.

\subsection{Discussion}

\subsubsection{Differences between the controllers}

These numerical results can be explained by the character of these controllers. The PID control with constant gains has a simple implementation and low computational cost, but it is linear. It cannot perform in nonlinear systems. The structure of the fuzzy P+ID controller is also very simple since it is constructed by replacing the proportional term in the conventional PID controller with a Mamdani FLS which has fuzzy rules based on expert knowledge. The Fuzzy P+ID controller, therefore, somehow improves the control performance. The MF-PID-FLC uses a TSK FLS based on extended subtractive clustering, which ensures the high precision of systematic approach so that the system performance is greatly improved. Consequently, the AMFFMC combines the advantages of the fuzzy P+ID control and the MF-PID-FLC and obviously, has the best performance with the least joint position and velocity tracking errors.

\subsubsection{The PID Gains}

The PID gains could greatly influence the control performance. If their values were well-chosen, the parallel system would move along the trajectory with smaller error. As shown in Section $\mathrm{V}(A)$, which compares the fuzzy P+ID control with the PID control, and the AMFFMC with the MF-PID-FLC, the systems with proportional gain, self-tuning fuzzy control exhibit less tracking error and better performance than those with fixed gains.

\subsubsection{The stability}


The proposed AMFFMC is a combination of the fuzzy P+ID controller for the feedback part of the system, and the TSK fuzzy controller for the feed-forward, nonlinear part. In this section, it is proven that the state vector of this closed loop system is always bounded when the desired trajectory is bounded. For the simplicity of the proof, the P+ID fuzzy controller is reduced to a $\mathrm{P}+\mathrm{D}$ fuzzy controller (i.e. the integral term is avoided). However, the proof could be extended in order to include the integral term by using the Lyapunov function. By avoiding the integral term of the P+ID controller, for any robot, the dynamics of the closed loop system can be expressed as follows:

$$
M(p) a+V(p, v) v+K_{p 0} p+K_{d} v=\Psi\left(a^{d}, v^{d}, p^{d}, e_{p}, e_{v}\right)
$$

where $M(p)$ is symmetric and positive definite matrix, $V(p, v)$ is a matrix that can be chosen such as that $\frac{1}{2} M(p)-V(p, v)$ is antisymmetric [38] and where

$$
\Psi\left(a^{d}, v^{d}, p^{d}, e_{p}, e_{v}\right)=\tau_{A N F I S}\left(a^{d}, v^{d}, p^{d}\right)+K_{p 0} p^{d}+K_{d} v^{d}+K_{p} u_{f u z z y}\left(e_{p}, e_{v}\right)
$$

is bounded since the desired trajectory is assumed to be bounded and the output of both fuzzy controllers are always bounded. Thanks to the bound of $\Psi$, it is known [38] that the state vector composed by the position $p$ and velocity $v$, is then bounded when the following system is asymptotically stable at $\mathbf{0}$ equilibrium point:

$$
M(p) a+V(p, v) v+K_{p 0} p+K_{d} v=0
$$

To prove the asymptotic stability of system (14), the following Lyapunov function, which is positive definite when $K_{p 0}$ is assumed to be symmetric and positive definite, is considered as candidate:

$$
v=\frac{1}{2} v^{T} M(p) v+\frac{1}{2} p^{T} K_{p 0} p
$$

The derivative of this function along the trajectory of the system is given by

$$
\dot{v}=\frac{1}{2} v^{T} M(p) v-v^{T} V(p, v) v-v^{T} K_{p 0} p-v^{T} K_{d} v+v^{T} K_{p 0} p
$$

Since $\frac{1}{2} \dot{M}(p)-V(p, v)$ is anti-symmetric, $\dot{v}=-v^{T} K_{d} v$ is semi negative definite when $\mathbf{K}_{d}$ is assumed to be symmetric and positive definite. Then, according to Lasalle theorem [38], the trajectory of the system will converge into the invariant set characterised by $v^{T} K_{d} v=0$. This set combined with the dynamics of system (14) implies that both position and velocity vectors will converge to zero, which implies that the system is asymptotically stable. The state vector of the complete system (12) composed by any robot combined with the double fuzzy controller is thus bounded.

\subsubsection{Limitation}

In this paper, the AMFFMC is in continuous-time form and the full nonlinear dynamics are used. Moreover, the control demands the measurement of the joint acceleration either by a rather expensive accelerometer, or by a complicated, or inaccurate and noisy calculation of acceleration from other measurements.

\subsection{Perspective}

To avoid the use of acceleration measurements and be closer to the real implementation, our further research will focus on a technique that employs position and velocity measurements by a model-free intelligent control in a discrete time domain. It is expected that the discrete-time version of the model-free intelligent control will not only have a flexible design and simple implementation but also will ensure good motion control quality and provide great robustness against discretization effects. 


\section{Conclusion}

This paper has proposed an accurate model-free fuzzy motion control (AMFFMC) for the control of a parallel robot. This AMFFMC combines a fuzzy P+ID controller and a TSK fuzzy controller. The fuzzy P+ID controller has a Mamdani fuzzy P term together with a conventional I plus D term to control the feedback part of the system. The TSK FLS controls the feedforward part of the system, ensures good control quality and small tracking error. The TSK FIS directly integrates on-line information from the nonlinear dynamics of the robot's joint displacement, velocity and acceleration in order to control motor torque without any complex computing of the robot's dynamic model. Moreover, the parametric searches for clustering parameters in extended subtractive- clustering ensure the accuracy of the system identification. The simulation results on a 4-bar parallel mechanism demonstrate the reliability of the AMFFMC in torque identification which, compared with several other intelligent controls, results in minimized joint position and velocity errors.

\section{References}

[1] Akbas K.: Application of Neural Networks to Modeling and Control of Parallel Manipulators. IN: Parallel Manipulators, New Developments, J. H. Ryu, Vienna, Austria: I-Tech Education and Publishing, 21-40 (2008)

[2] AL-Saedi M., Wu H. and Handroos H.: ANFIS and Fuzzy Tuning of PID Controller for Trajectory Tracking of a Flexible Hydraulically Driven Parallel Robot Machine, Journal of Automation and Control Engineering, 1(3), 70-77 (2013)

[3] Astrom K. J., Hang C. C., Persson P., and Ho W. K., Towards intelligent PID control, Automatica, 28(1) 1-9 (1992)

[4] Chang P. \& Jung P.: A systematic method for gain selection of robust PID control for nonlinear plants of secondorder controller canonical form. IEEE Transactions on Control Systems Technology, 17, 473-483 (2009)

[5] Chen W. H., Yang J., Guo L. \& Li, S.: Disturbance-Observer-Based Control and Related Methods - An Overview, IEEE Transactions on Industrial Electronics, 63(2), 1083-1095 (2016)

[6] Chiu S. L.: Fuzzy model identification based on cluster estimation, Journal on International Fuzzy Systems, 2, 267-278 (1994)

[7] Demirli, K. Cheng S. X., Muthukumaran P.: Subtractive clustering based modeling of job sequencing with parametric search. Fuzzy Sets and Systems. 137(2), 235-270 (2003)

[8] Fazzolari M., Alcala R., Nojima Y., Ishibuchi H. \& Herrera F.: A review of the application of multiobjective evolutionary fuzzy systems: Current status and further directions, IEEE Transactions on Fuzzy Systems, 21(1), 46-65 (2013)

[9] Feng, G.: A Survey on Analysis and Design of Model-Based Fuzzy Control Systems, IEEE Transaction on Fuzzy Systems,_14(5) 676-697 (2006)

[10] Fliess M. and Join C.: Model-free control, International Journal of Control, 86(12) 2228-2252 (2013).

[11] Formentin S., de Filippi P., Corno M., Tanelli M.\& Savaresi, S.: Data-driven design of braking control systems. IEEE Transactions on Control Systems Technology, 21,186-193 (2013)

[12] Ho, W. K., Hong Y, Hansson A, Hjalmarsson, H.and. Deng, J. W.: Relay auto-tuning of PID controllers using iterative feedback tuning, Automatica 39 (1), 149-157 (2003)

[13] Jang J.-S. R.: ANFIS: Adaptive-Network-based Fuzzy Inference Systems, IEEE Transactions on Systems, Man, and Cybernetics, 23(3), 665-685 (1993)

[14] Keel L. H. \& Bhattacharya S. P.: Controller synthesis free of analytical models: Three term controllers. IEEE Transactions on Automatic Control, 53, 1353-1369 (2008)

[15] Li, W.: Design of a Hybrid Fuzzy Logic Proportional Plus Conventional Integral-Derivative Controller, IEEE trans. On Fuzzy Systems, 6(4), 449-463 (1998)

[16] Lin, C. M. \& Li, H. Y.: Dynamic Petri Fuzzy Cerebellar Model Articulation Controller Design for a Magnetic Levitation System and a Two-Axis Linear Piezoelectric Ceramic Motor Drive System, IEEE Transactions on Control Systems Technology, 23(2), 693 - 699 (2015)

[17] Mahfouf, M., Abbod M. F. \& Linkens, D. A.: Online elicitation of Mamdani-type fuzzy rules via TSK-based generalized predictive control, IEEE Transactions on Systems, Man, and Cybernetics, Part B: Cybernetics, 33(3), 465 - 475 (2003)

[18] Mamdani E. H. and Assilian, S.: Applications of Fuzzy Algorithms for Control of Simple Dynamic Plant, Proc. Inst. Elec. Eng., 121, 1585-1588 (1974)

[19] Mendel. J. Hagras H., Tan W., Melek, W. \& Ying H., Introduction to type-2 fuzzy logic control, theory and applications. Wiley-IEEE Press, 2014.

[20] Milanes, V. Villagra, J. , Perez J. \& Gonzalez C.: Low speed longitudinal controllers for mass-produced cars: A comparative study. IEEE Transactions on Industrial Electronics, 59, 620-628 (2012)

[21] Morales R., Feliu V.\& Sira-Ramirez, H.: Nonlinear control for magnetic levitation systems based on fast online algebraic identification of the input gain". IEEE Transactions on Control Systems Technology, 19, 757-77 (2011) 
[22] Pedrycz, W.: Identification in Fuzzy Systems, IEEE Transaction on Fuzzy Systems, 14, 361-366 (1984)

[23] Precup, R.E., Hellendo H.: A survey on industrial applications of fuzzy control, Computers in Industry, 62(3), 213-226 (2011)

[24] Qi, Z. Mcinroy J. E. and Jafari F.: Trajectory Tracking with Parallel Robots Using Low Chattering Fuzzy Sliding Mode Controller, Journal of Intelligent and Robotic Systems. 48(3), 333-356 (2007)

[25] Rad, A. B., Chan, P. T., and Mok, C. K.: An online learning fuzzy controller, IEEE Transactions on Industrial Electronics, 50(5), 1016 - 1021 (2003)

[26] Ren Q., Baron L., Balazinski M., Jemielniak K.: TSK Fuzzy Modeling for Tool Wear Condition in Turning Processes: An Experimental Study, Engineering Applications of Artificial Intelligence, 24(2), 260-265 (2011)

[27] Ren Q., Balazinski M., Jemielniak K., Baron L., and Achiche S.: Experimental and Fuzzy Modeling Analysis on Dynamic Cutting Force in Micro-milling, Soft Computing, 17, 1687-1697 (2013)

[28] Ren Q. and Bigras P.: Model-Free Adaptive Neural Fuzzy Feed Forward Torque Control for Nonlinear Parallel Mechanism. In: IEEE/ASME International Conference on Advanced Intelligent Mechatronics, Busan, Korea, 1043-1048 (2015)

[29] Ren Q., Baron L., Balazinski M., Jemielniak K., Botez R. and Achiche S.: Type-2 Fuzzy Tool Condition Monitoring System Based on Acoustic Emission in Micromilling. Information Science. 255, 121-134 (2014)

[30] Sugeno M. \& Kang, G.: Fuzzy Modeling and Control of Multilayer Incinerator, Fuzzy Sets and Systems, 18, 329-346 (1986)

[31] Sun Y. L. \& Er. M. J.: Hybrid fuzzy control of robotics systems, IEEE Transactions on Fuzzy Systems, 12(6), 755-765 (2004)

[32] Syafiie S. Tadeo F., Martinez E. \& Alvarez T.: Model free control based on reinforcement learning for a wastewater treatment problem. Applied Soft Computing, 1, 73-82 (2011)

[33] Takagi T. and Sugeno, M.: Fuzzy Identification of Systems and Its Applications to Modeling and Control, IEEE Transaction on Systems, Man, and Cybernetics, 15(1), 116-132 (1985)

[34] Tong, R. M.: The Construction and Evaluations of Fuzzy Models, in: M.M. Gupta, R.K. Rewgade, R.R. Yager (Eds.), Advances in Fuzzy Set Theory and Applications, North-Holland, Amsterdam, 559-576 (1979)

[35] Villagra J. \& Herrero-Perez D.: A comparison of control techniques for robust docking maneuvers for an AVG. IEEE Transactions on Control Systems Technology, 20, 1116-1123 (2012)

[36] Wang F. Y., Liu, D.: Networked control systems: theory and applications, Springer Verlag, 2008.

[37] Yager R. R. and Filev P.: Essentials of Fuzzy Modeling and Control. New York, NY: John Wiley, 1994

[38] Zhihua Qu and Darren M. Dawson, Robust Tracking Control of Robot Manipulators, IEEE Press, 1996, 233 pages.

[39] Zi, B. Y. Duan, J., Du L. and Bao H.: Dynamic modeling and active control of a cable-suspended parallel robot, Mechatronics, 18(1), 1-12 (2008) 